\title{
Evolution of a neglected ureteral stent
}

\author{
Vikrampal Bhatti ${ }^{1} \cdot$ Abhilash Koratala ${ }^{1}$
}

Received: 8 December 2016/ Accepted: 6 January 2017/Published online: 16 January 2017

(C) SIMI 2017

A 27-year-old woman with a history of recurrent nephrolithiasis presented with right flank and suprapubic pain for 4-day duration. She also complained of fever and chills. She had a right ureteral double J stent placed 3 years prior to presentation for right-sided urolithiasis. The stent was not exchanged or removed during this period as she was lost to follow-up with the urologist. She had a fever of $38.2{ }^{\circ} \mathrm{C}$, and other vital signs were normal. Physical examination was unremarkable except for tenderness in the right costo-vertebral angle. Urinalysis revealed about 1600 white blood cells and 400 red blood cells per high power field. She was admitted to the hospital, and started on intravenous antibiotics for pyelonephritis. Later, urine culture grew more than $100,000 \mathrm{col} / \mathrm{ml}$ Klebsiella pneumoniae. An abdominal computed tomography (CT) scan was obtained on day 3 of admission because of persistent abdominal pain and fever, to rule out possible perinephric abscess. It showed right pelvi-ureteral junction nephrolithiasis with an encrusted right ureteral stent, and a large bladder stone (Figs. 1, 2). Cystoscopy showed a large, free floating $\sim 6 \mathrm{~cm}$ spherical stone within the bladder. She subsequently underwent cystolithalopaxy, laser lithotripsy, right percutaneous nephrolithotomy and removal of the distal retained stent. Most of the stone burden including the bladder and uretero-pelvic junction stones was removed. Stone analysis revealed carbonate apatite and magnesium ammonium phosphate hexahydrate.

Ureteral stents have become the mainstay of treatment of ureteral obstruction. Stent migration, fragmentation and encrustation are the common late complications associated with them [1]. Ureteric stent encrustation is often associated with a stent indwelling time of more than 12 weeks [2], alkaline urine, urinary tract infections, poor compliance and congenital renal anomalies [3]. The underlying mechanism of encrustation is that the urinary proteins and bacterial products adhere to the stent surface leading to a biofilm formation that in turn, leads to calcium and oxalate precipitation. The presence of a foreign body (that is, the lower portion of the ureteral stent) in the urinary bladder increases the risk of recurrent urinary tract infections, thereby biofilm formation and bladder stone formation [4]. The most common stones associated with encrustation are calcium oxalate $(69.1 \%)$ and carbapatite (15.5\%) [5]. Management of encrusted stents remains challenging, and

Abhilash Koratala

akoratsla@ufl.edu

1 Division of Nephrology, Hypertension and Renal Transplantation, University of Florida, P.O. Box 100224, Gainesville, FL 32610, USA 


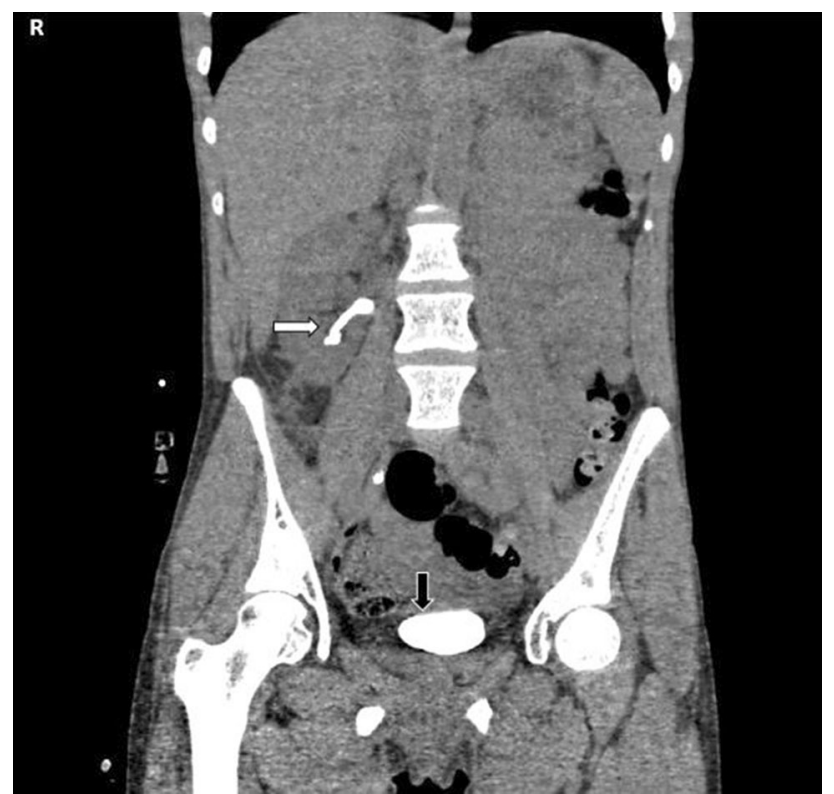

Fig. 1 CT scan of the abdomen without contrast showing encrusted right ureteral stent (white arrow) and a large bladder stone measuring about $6 \mathrm{~cm}$ (black arrow)

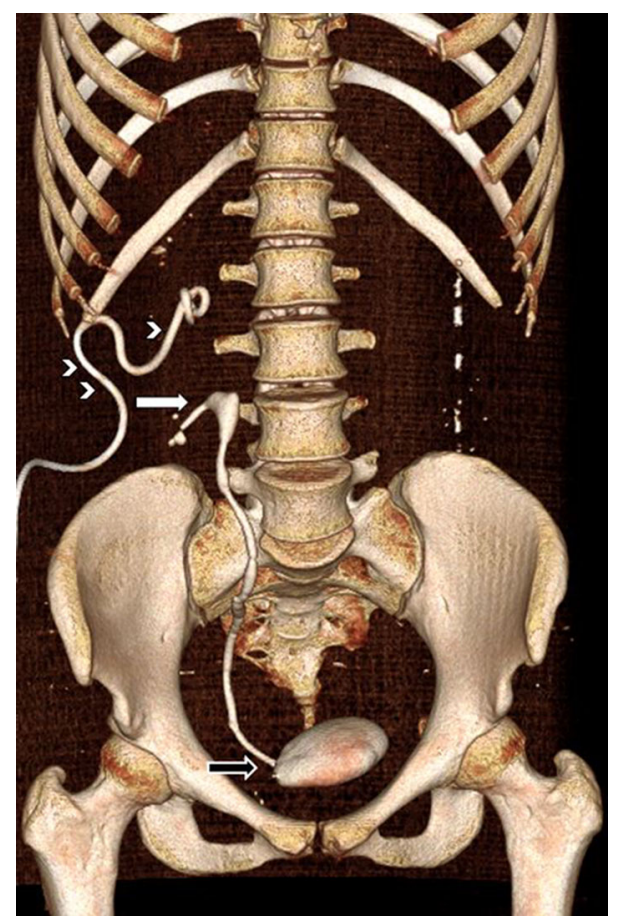

Fig. 2 3D reconstructed CT scan image of the encrusted right nephro-ureteral stent (white arrow) and bladder stone (black arrow). The image also shows a nephrostomy tube placed during the urologic procedure, indicated with chevrons timely removal or exchange of ureteral catheters should minimize risk of stent calcification. Patient education and maintenance of a computerized registry to prevent 'forgotten stents' may play a key role in prevention.

\section{Compliance with ethical standards}

Conflict of interest The authors declare that they have no conflict of interest.

Statement of human and animal rights All procedures followed were in accordance with the ethical standards of the responsible committee on human experimentation and with the Helsinki Declaration of 1975, as revised in 2013. No animals were used during the study.

Informed consent Informed consent was obtained from the patient for publication of this study.

\section{References}

1. Damiano O, Esposito C, De Sio M, Autorino R et al (2002) Early and late complications of double pigtail ureteral stent. Urol Int 69(2):136-140

2. ElFaqih S, Shamsuddin A, Chakrabarti A et al (1995) Polyurethane internal urethral stents in treatment of stone patients: morbidity related to indwelling times. J Urol 153:1817-1820

3. Singh I, Gupta N, Hemal A et al (2001) Severely encrusted polyurethane ureteral stents: management and analysis of potential risk factors. Urology 58(4):526-531

4. Bidnur S, Huynh M, Hoag N et al (2016) An indwelling ureteral stent forgotten for over 12 years. J Endourol Case Rep 2(1):135-137

5. Rouprêt M, Daudon M, Hupertan V et al (2005) Can ureteral stent encrustation analysis predict urinary stone composition? Urology 66(2):246-251 